\title{
Resolution Enhancement for Tomographical Images of a Full-Field OCT System
}

\author{
Shoude Chang ${ }^{*}, 1$, Xianyang $\mathrm{Cai}^{2}$ and Costel Flueraru ${ }^{1}$ \\ ${ }^{I}$ Imaging Devices Group, Institute for Microstructural Sciences, National Research Council, K1A 0R6, Canada \\ ${ }^{2}$ SENSATA Technologies, USA
}

\begin{abstract}
In this paper a new method is proposed to enhance the resolution of tomographical images of a full-field optical coherence tomography (OCT) system without improving the bandwidth of light source. Theoretic study and simulation indicate that the method improves the system resolution and quality of retrieved tomographic images for the multilayer information carrier. Experiment results are also given in support of the proposed method.
\end{abstract}

Keywords: Image processing, image enhancement, cross-sectional image extraction, optical coherent tomography.

\section{OCT SYSTEM FOR IMAGE RETRIEVAL FROM A MULTI-LAYER OBJECT}

Optical coherence tomography (OCT) is a powerful imaging technology for producing high-resolution crosssectional images of the internal microstructure of materials and biological samples $[1,2]$. OCT systems applied to image retrieval from multi-layer information carrier were reported in $[3,4]$. The axial resolution of an OCT system is conventionally defined by coherent length $L c$, or coherence gate, of the light source. If the separation between two layers is within $L c$, image retrieval from the interference pattern becomes extremely difficult for the conventional method.

\section{THREE-STEP PHASE SHIFT METHOD}

Phase shift interferometry is known since three decades and many methods were proposed to improve the resolution and accuracy of the interferometry [5-8]. However, most of them are aimed to extract the phase information from the interference fringes obtained. OCT works in a different way. It targets on extracting the cross-sectional intensity image at each layer. The interference fringes presented have to be removed completely $[3,4]$.

Suppose the object beam is consist of two beams, $A_{l}=O_{1} \exp \left(\mathrm{j} \varphi_{1}\right)$ at the first layer and $A_{2}=O_{2} \exp \left(\mathrm{j} \varphi_{2}\right)$ at the second layer. $O_{1}$ and $O_{2}$ are image patterns, and $\varphi_{1}$ and $\varphi_{2}$ are phases for both layers. For a partial coherence light source, the contrast of the interference fringe by a layer is dependent on the location of the layer on the profile of interference signal, as shown in Fig. (1). Suppose the degrees of coherence of the first layer and the second layer with reference are $C_{1}$ and $C_{2}$, respectively, the interferogram between the object beam and reference beam can be expressed as,

$I=I_{0}+I_{M}+2 O_{1} C_{1} \cos \left(\varphi_{1}\right)+2 O_{2} C_{2} R \cos \left(\varphi_{2}\right)$,

where $R$ is the amplitude of reference beam, $I_{0}$ is a non-

*Address correspondence to this author at the Imaging Devices Group, Institute for Microstructural Sciences, National Research Council, K1A 0R6, Canada; Tel: 613-9909716; Fax: 613-954-3338;

E-mail: shoude.chang@nrc.ca coherent background image and $I_{M}$ is the mutual interference between two layers. It is noted that $I_{M}$ is unchanged when coherent gate is set on different positions and this term can be considered as a constant background. The phase differences $\varphi_{1}$ and $\varphi_{2}$ are variable with the setting of coherent gate.

To obtain the information from the layers, $O_{1}$ and $O_{2}$ need to be solved from Equation (1). A three-step method to extract the image pattern from the interference pattern is developed. For simplification, the reference is supposed to be a uniform beam and $R=1$. In the first step, the coherent gate is set on the first layer of info chip, e.g., $C_{l}=1$.

Three interferograms are then recorded as follow,

$$
\begin{aligned}
& G_{11}=I_{b}+O_{1} \cos \left(\varphi_{11}\right)+C_{2} O_{2} \cos \left(\varphi_{21}\right) \\
& G_{12}=I_{b}+O_{1} \sin \left(\varphi_{11}\right)+C_{2} O_{2} \sin \left(\varphi_{21}\right) \\
& G_{13}=I_{b}
\end{aligned}
$$

Equation (2) is the same fringe pattern as Equation (3) except a phase shift of $\pi / 2$ for the reference beam. $I_{b}=I_{0}+$ $I_{M}$, is just a non-interference background image which correspond to the signal obtained from the reference mirror being blocked. $\varphi_{11}$ and $\varphi_{21}$ represent the phases of first layer and second layer respectively. From above equations, following expression can be derived as,

$$
O_{10}^{2}=O_{1}^{2}+\left(C_{2} O_{2}\right)^{2}+4 O_{1} C_{2} O_{2} \cos \left(\Delta \varphi_{1}\right)
$$

where $O_{10}=\left[\left(G_{11}-G_{13}\right)^{2}+\left(G_{12}-G_{13}\right)^{2}\right]^{1 / 2}$ is actually a solution for the first layer image when the second layer contribution to the interference signal is zero. $\Delta \varphi_{1}=\varphi_{11}-\varphi_{21}$ is the phase difference between two layers.

Then the coherent gate is moved to second layer such that $C_{2}=1$ and another set of three images are captured as,

$$
\begin{aligned}
& G_{21}=I_{b}+C_{1} O_{1} \cos \left(\varphi_{21}\right)+O_{2} \cos \left(\varphi_{22}\right) \\
& G_{22}=I_{b}+C_{1} O_{1} \sin \left(\varphi_{21}\right)+O_{2} \sin \left(\varphi_{22}\right) \\
& G_{23}=I_{b}
\end{aligned}
$$




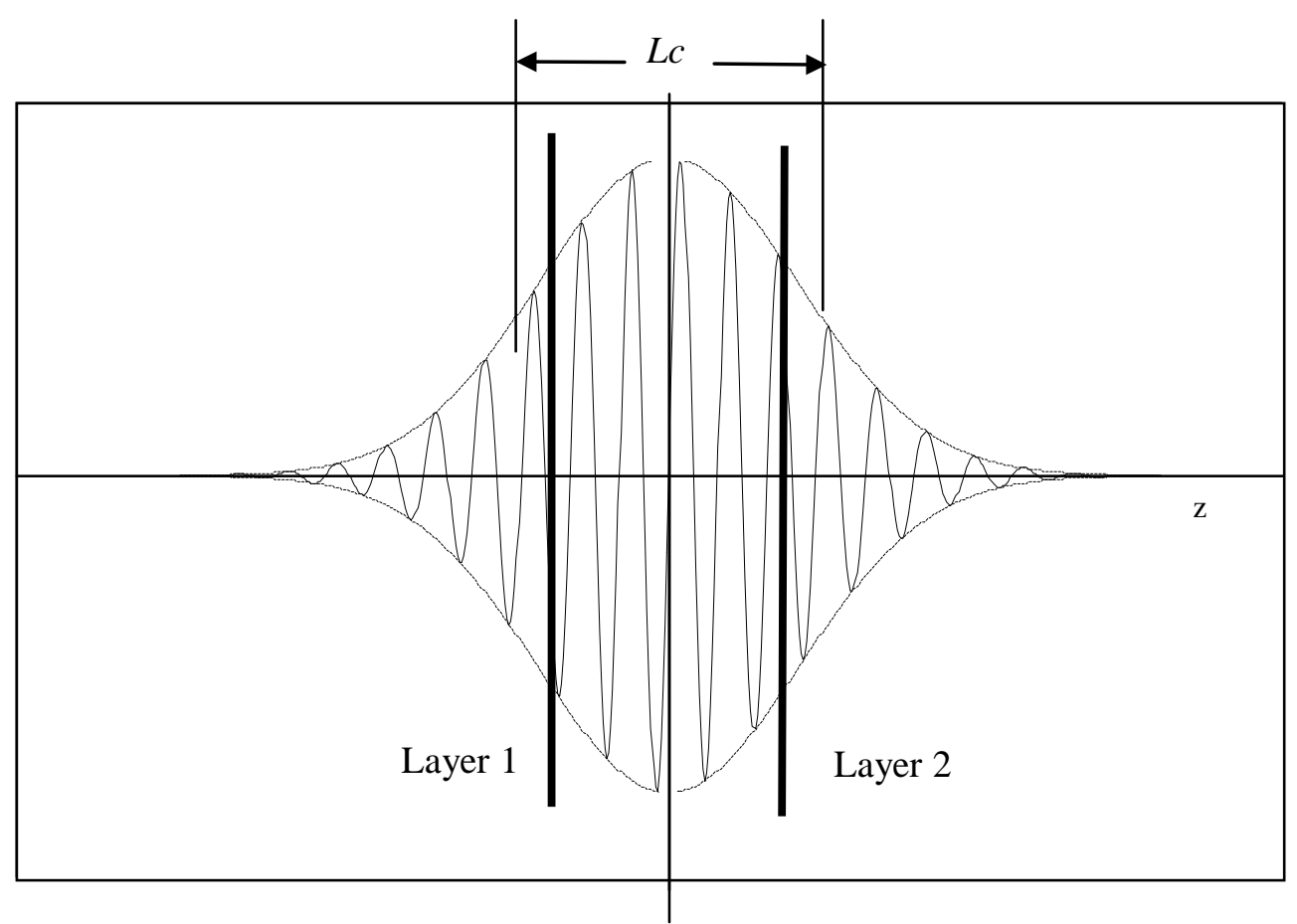

Fig. (1). Intensity variation of interference signal with axial position shift.

where $\varphi_{21}$ and $\varphi_{22}$ represent the phases of first layer and second layer when coherent gate is on the second layer. Similarly, an image pattern for the second layer can be derived as,

$$
O_{20}^{2}=O_{2}^{2}+\left(C_{1} O_{1}\right)^{2}+4 O_{1} C_{1} O_{2} \cos \left(\Delta \varphi_{2}\right)
$$

where $O_{20}=\left[\left(G_{21}-G_{23}\right)^{2}+\left(G_{22}-G_{23}\right)^{2}\right]^{1 / 2}$ is a solution for the second layer image when the first layer contribution to the interference signal is zero. For a partial coherent source, the envelop of the interference signal shown in Fig. (1) is symmetrical around its peak position. Therefore coefficients of coherence in two steps should be the same, e.g. $C_{I}=C_{2}=C$.

In third step, the coherent gate is set between layer 1 and layer 2, so that the two layers have the same degree of coherence and their fringes have the same contrast. Then, A similarly equation can be derived as,

$O_{30}^{2}=C_{3}^{2} O_{1}^{2}+C_{3}^{2} O_{2}^{2}+4 C_{3}^{2} O_{1} O_{2} \cos \left(\Delta \varphi_{3}\right)$

where $O_{30}=\left[\left(G_{31}-G_{33}\right)^{2}+\left(G_{32}-G_{33}\right)^{2}\right]^{1 / 2}$. Obviously, $\Delta \varphi_{1=} \Delta \varphi_{2=}$ $\Delta \varphi_{3}$ if the reference keep uniform phase in all the three steps, and this condition can be easily met in experiment. From Equation (5), (9) and (10), the image patterns of both layers can be solved as,

$$
\begin{aligned}
& O_{1}=\left[\frac{O_{10}^{2} / C-O_{30}^{2} / C_{3}^{2}-\left(O_{10}^{2}-O_{20}^{2}\right) /(1+C)}{1 / C+C-2}\right]^{1 / 2} \\
& O_{2}=\left[O_{1}^{2}+\left(O_{10}^{2}-O_{20}^{2}\right) /\left(1-C^{2}\right)\right]^{1 / 2}
\end{aligned}
$$

The above equations give a simple solution for the images on two layers. These solutions indicate that the knowledge for the values of $C$ and $C_{3}$ are very important for this algorithm. These values are dependent on setting of the coherent gate and separation between layers. In practical, the initial values of these parameters should be obtained experimentally and accurate values are then obtained through an iteration process for best image quality.

\section{SIMULATION AND EXPERIMENT AND RESULTS}

The three-step method provides an approach to improve the axial resolution for the OCT system. To prove the efficiency of the method, a simple simulation is conducted and the results are given in Fig. (2). The first layer image is a word of "OCT" and second layer one is a word "NRC". Separation between the layers is $20 \mu \mathrm{m}$. The values of coherent coefficient $C$ and $C_{3}$ is set to be 0.6 and 0.88 , respectively. Those numbers are obtained experimentally through some iteration to minimize the fringe contrast in final image. Fig. (2a, b) are retrieved images by a conventional algorithm. The two images show strong ghost images from other layers and a parasite fringes. Fig. (2c, d) give retrieved images using the new proposed three-step method. Both ghost images from other layers and parasite fringes are eliminated.

The experimental setup is based on a full-field OCT system (Fig. 3). It is a Michelson interferometer integrated with computer controlled motion-vision system. The output of the superluminescent laser diode (SLD) is collimated by lens $\mathrm{LS}_{1}$, and then divided into the reference and sample arms by a non-polarized BS. A neutral density filter (NDF) is used to adjust the intensity of the reference beam reflected from the reference mirror (RM). Lens $\mathrm{LS}_{2}$ images the info-chip on the CCD camera. The least thickness of each layer that can be resolved, $L c$, is determined by the central wavelength $\lambda$ and spectrum width $\Delta \lambda$ of SLD used: $L c=0.44 \lambda^{2} / \Delta \lambda$. In our system, as $\lambda$ and $\Delta \lambda$ are $830 \mathrm{~nm}, 15 \mathrm{~nm}$, respectively, the depth resolution is $20 \mu \mathrm{m}$. The lateral resolution of an OCT device is basically determined by the lens $\mathrm{LS}_{2}$ and resolution 


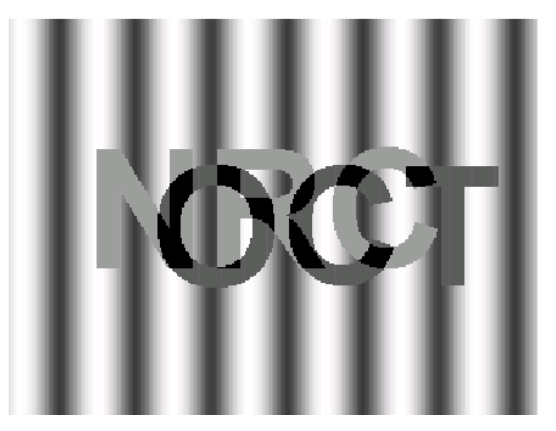

(a)

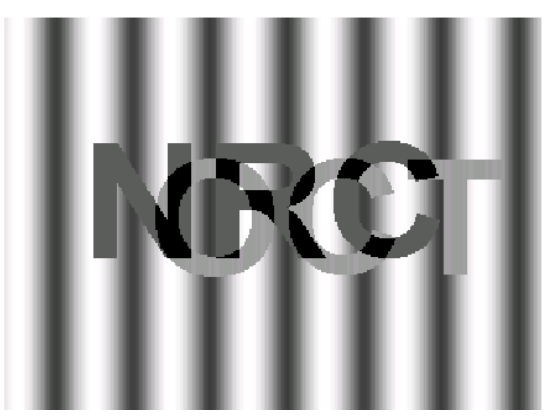

(b)

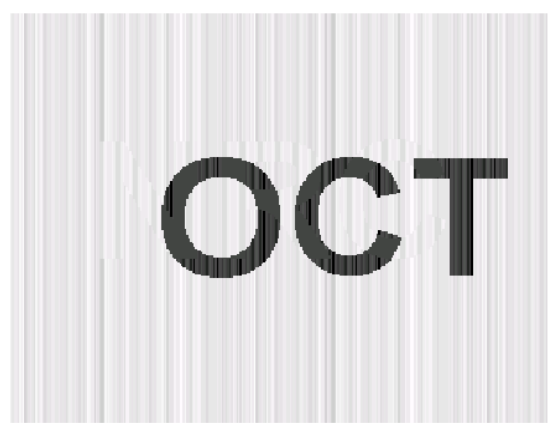

(c)

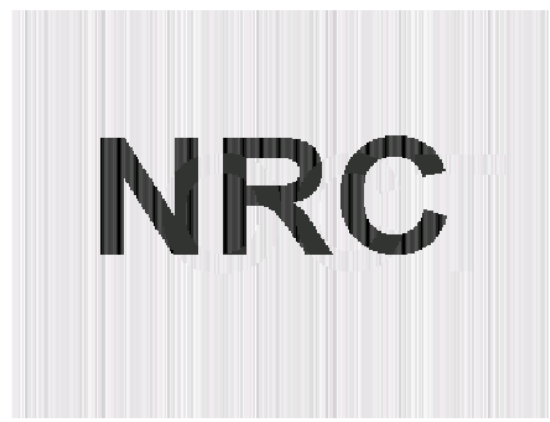

(d)

Fig. (2). Simulation results of three-step method. (a, b): restored image with conventional method; (c, d): restored images with three-step method.

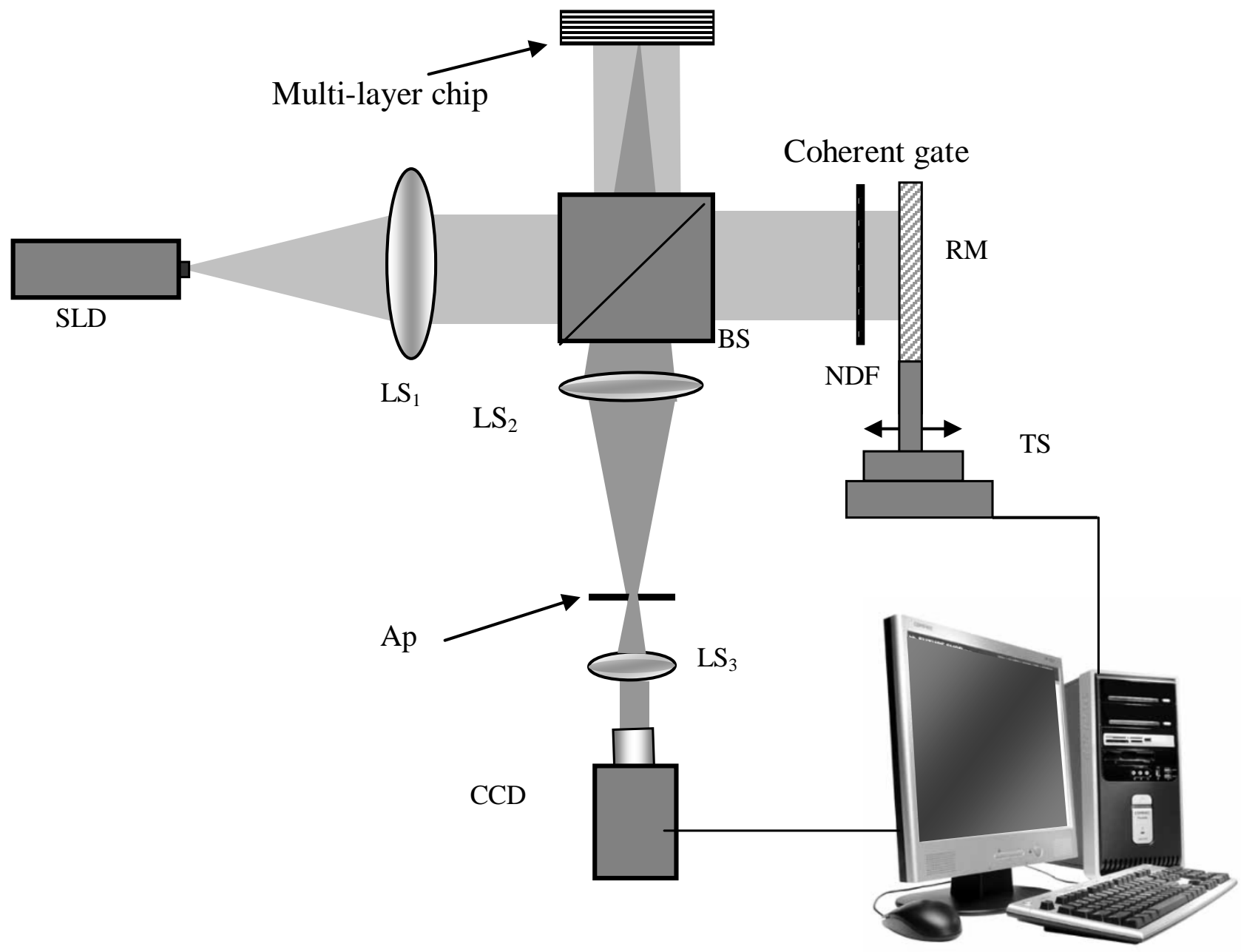

Fig. (3). Full-field OCT system used in the experiment. 

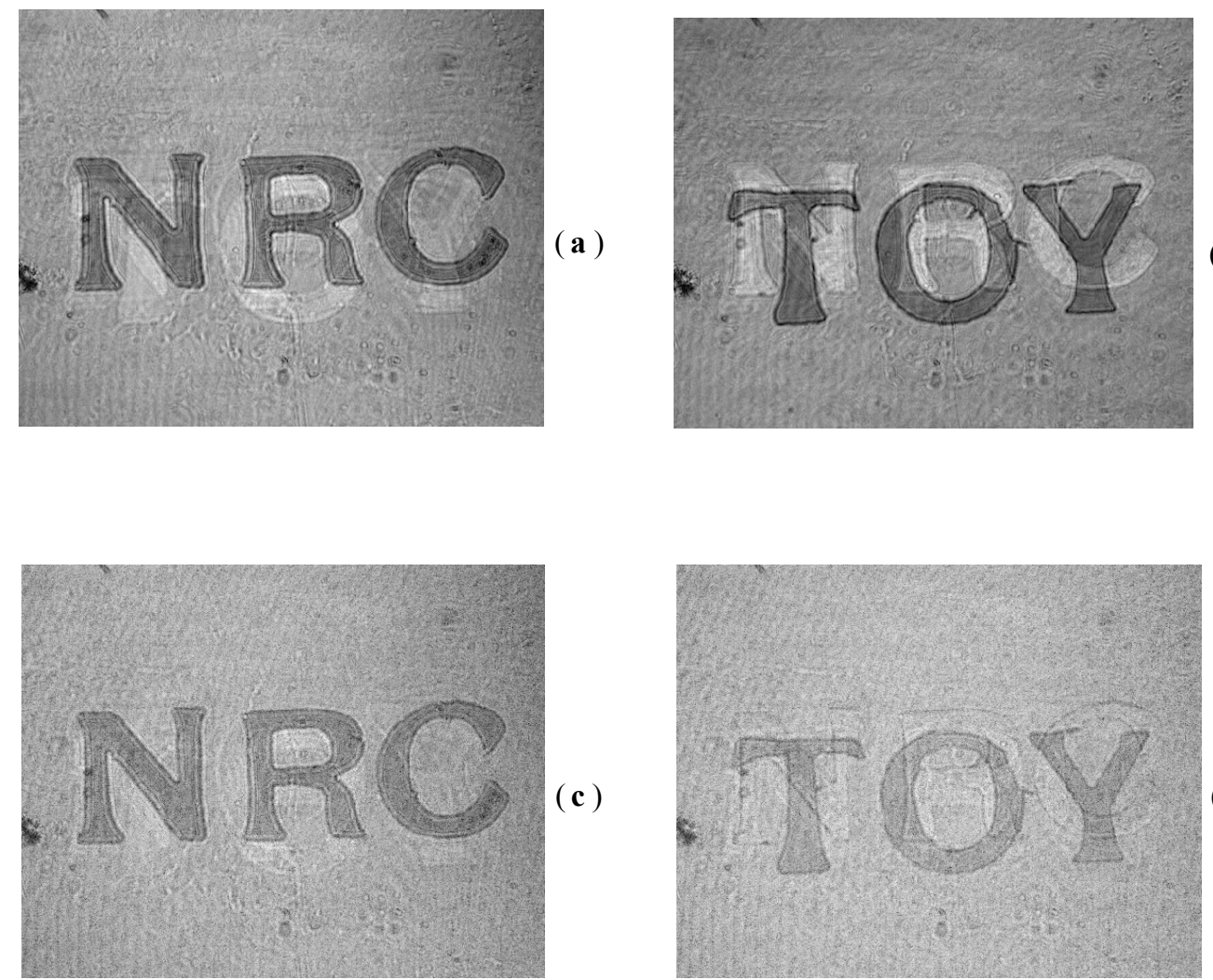

Fig. (4). Experimental results of three-step method. (a, b): restored image with conventional method. (c, d): restored images with three-step method. These images have a physical size of $15 \mathrm{~mm}$ by $15 \mathrm{~mm}$.

of CCD camera. In our experimental setup, the viewing area of the sample is $20 \mathrm{~mm}$ by $20 \mathrm{~mm}$ and the CCD has a resolution of $1024 \times 1024$ pixels, the lateral resolution of the system is then $20 \mu \mathrm{m}$. The differences of phase and reflective index between the ink and surface of the layer make the 2D information distinguishable. The sample to be tested is a two-glass plate with coated text patterns on. The coated surface has a $15 \mathrm{~mm}$ by $15 \mathrm{~mm}$ area and is air-gapped by about $20 \mu \mathrm{m}$. The OCT system was first characterized by using a mirror to obtain contrast variation of interference fringe with axial shift. From this figure, the initial values of $C$ and $C_{3}$ are determined. Fig. (4) is the tomographic images restored by conventional method. It can be seen the ghost image from other layer is added on the interest images, as predicated in theory. Fig. (4a, b) show the tomographic images retrieved by proposed algorithm with $C=0.1$ and $C_{3}=0.55$. Let the resolution corresponding to $C$ and $C_{3}$ be $\mathrm{Z}_{1}$ and $\mathrm{Z}_{2}$, respectively, and the coherence gate has a Gaussian distribution, it is easy to find that $\left(Z_{1}-Z_{2}\right) / Z_{2}=0.574$, i.e., the axial resolution has been improved about $57 \%$. In this experiment, the depth resolution is enhanced from $20 \mu \mathrm{m}$ to $11.4 \mu \mathrm{m}$.

\section{CONCLUSION AND DISCUSSION}

For conventional OCT system, to achieve the higher axial resolution, a broader band light sources has to be used. Currently, the bandwidth of the OCT light source is less than $100 \mathrm{~nm}$. With the new method presented in this paper we can achieve a relatively high resolution without limited by the bandwidth or coherence length of the light source. This is particularly useful to the full-filed OCT systems with artificial multiple-layer samples whose material and thickness can be carefully selected. Both theoretical simulation and experiment verification proved the efficiency of the method. Although the theoretical deduction and experimental verification were conducted based on a two-layer info chip, it is not limited to two-layer information retrieval. The principle could be expanded to multilayer samples or even other type of full-field OCT system to improve the resolution and quality of tomographic images.

However, there are some drawbacks for the proposed three steps method. Firstly, this method is specifically designed for the applications associated with multilayer parallel structures. As for the bio-sample, it may still need further improvement and modification. Another one is the higher requirement for the motion control system. Particularly, when the system has relatively higher depth resolution, for example $5 \mu \mathrm{m}$, the mechanical control becomes more accurate, which increases the cost of the system and also reduces the speed of processing time. In addition, this method is based on a time-domain OCT system, it can not be applied to the Fourier-domain OCT, where no motion part is needed.

\section{REFERENCES}

[1] Huang D, Swanson EA, Lin CP, et al. Optical coherence tomography. Science 1991; 254: 1178-81.

[2] Fercher AF, Drexler W, Hitzenberger CK, Lasser T. Optical coherence tomography-principles and applications. Rep Prog Phys 2003; 66: 239-303.

[3] Chang S, Liu X, Cai X, Grover CP. Full-field optical coherence tomography and its application to multiple-layer $2 \mathrm{D}$ information retrieving. Opt Commun 2005; 246: 579-85. 
[4] Chang S, Cai X, Flueraru C. Image enhancement for multilayer information retrieval using full-field optical coherence tomography. Appl Opt 2006; 45(23): 5967-75.

[5] Guo CS, Rong ZY, He JL, Wang HT, Cai LZ, Wang YR. Determination of global phase shifts between interferograms by use of an energy-minimum algorithm. Appl Opt 2003; 42: 6514-9.

[6] Langoju R, Patil A, Rastogi P. Super-resolution fourier transform method in phase shifting interferometry. Opt Express 2005; 13: 7160-73.
[7] Chang S, Cai X, Flueraru C. An Efficient algorithm used for fullfield optical coherence tomography. Opt Lasers Eng 2007; 45: 1170-6.

[8] Gao P, Yao B, Han J, Chen L, Wang Y, Lei M. Phase and amplitude reconstruction from a single carrier-frequency interferogram without phase unwrapping. Appl Opt 2008; 47(15): 2760-6.

Received: February 3, 2009

Revised: March 4, 2009

Accepted: March 12, 2009

(C) Chang et al.; Licensee Bentham Open.

This is an open access article licensed under the terms of the Creative Commons Attribution Non-Commercial License (http://creativecommons.org/licenses/by$\mathrm{nc} / 3.0 /$ ) which permits unrestricted, non-commercial use, distribution and reproduction in any medium, provided the work is properly cited. 